\title{
PSYCHIATRIC ASPECTS OF SOMATIC IMMUNITY DIFFERENTIAL INCIDENCE OF PHYSICAL DISEASE IN THE HISTORIES OF PSYCHIATRIC PATIENTS
}

\author{
BY \\ J. W. LOVETT DOUST \\ From the Department of Psychiatry, University of London Institute of Psychiatry, \\ Maudsley Hospital
}

There have been few studies in the past of the incidence of physical disease in the mentally ill relative to that of the general population. Many writers have pointed to the concurrent or subsequent development of such disorders as pulmonary tuberculosis or enteric fever during the sojourn of psychiatric patients in mental hospitals, and there are a considerable number of reports from physicians concerning the intercurrence of mental symptoms in a variety of infections, traumata, tumours, intoxications, and other stressful experiences. This latter literature has been referred to in another communication (Lovett Doust, 1952), and an assessment of physical disorders present in patients admitted to a mental hospital has been made by Phillips (1937). In addition to these observations, a small number of studies have been concerned with the relative frequency of physical complaints in neurotically disturbed patients (Eysenck, 1947), with the length of convalescence of psychiatric patients afflicted by various bodily illnesses (Brodman and others, 1947a), and with the increased tendency of apparently mentally healthy patients hospitalize̊d for physical illness to show neurotic scores on a psychological test (Cornell Service Index) as compared with other subjects who had not broken down (Brodman and others, 1947b). The current interest in the general adaptation syndrome of Selye (1950), as it affects the capacity for homeostatic adaptation of mentally-ill patients (Hoagland and Pincus, 1950) has been extended into the field of immunity, especially by the work of Dougherty and others (1945) on changing lymphocyte counts, these cells being thought to be concerned in immunity production (White and Dougherty, 1946).

It is clinically not infrequent that both the general physician and the psychiatrist have the opportunity of witnessing what may be called the alternation of psychiatric and somatic disease in the course of the illness of an individual patient. This alternation may take the form, for example, of a confusional episode associated with pneumonia and occur before, during the height of, or after the pyrexia; it may be observed in the form of a psychotic reaction, similar in symptomatology and appearance to an "idiopathic" psychiatric syndrome, perhaps as the sequela of an attack of influenza or of an apparently normal pregnancy and labour; or it may occur as a somatic illness during the investigation and treatment of a mental disturbance and present as bronchial asthma, rheumatoid arthritis, etc. (Appel and Rosen, 1950).

The present paper stems from the clinical impression in the wards of a psychiatric 
hospital that the somatic illness history loading of the patients seen there appeared heavier than that of a control group of healthy subjects. This impression led to an endeavour to assess the known factors concerned in the development of immunity responses and it was surprising to the writer that these factors are both so few and so relatively unimportant. Apart from the dose of the infecting organism, most of them seem to be accounted for by age, sex, race, diet, state of nutrition, climate, and seasonal variation, together with a group of " $X$ " factors relating to individual susceptibility and resistance about which little is known. It is of some psychiatric interest that one of these " $X$ " factors may be that of fatigue, since it has been shown that susceptibility is increased when fatigue is present (Boycott and Price-Jones, 1926). The evidence however suggests that fatigue tended to act as a factor reactivating existing infection rather than as one predisposing to new infection. Perhaps the most obvious of the known factors listed above is state of nutrition, yet even here (as, for example, the investigations of Gell (1948) and of Balch (1950) have shown), it requires the most extreme forms of under-nutrition to effect a significant change in the ability to form antibodies against inoculated antigens and only pre-mortal states of undernourishment can materially alter the individual threshold. Various components of the diet have been implicated but none has been shown to be of general importance; indeed the evidence for indicting a number of vitamins appears to be both confusing and extremely flimsy. As Wilson and Miles (1950) point out, the vitamin deficiencies which seem to have some definite effect in this respect are avitaminoses $\mathrm{A}$ and $\mathrm{C}$. Of these the former appears to be of major importance only during the period of rapid bodily growth, while both deficiencies seem to influence resistance only when the lack of the factor is a gross one. Furthermore, apart from Gell's work, most investigations have been done on animals, in whom the whole pattern of individual response to human infections is so often different from that of man.

In recent years evidence has begun to accumulate in a rather different direction towards the elucidation of certain hitherto neglected factors concerned in the predisposition to infective and other disease processes. An orientation of medicine, loosely called the "psychosomatic" approach, based on a growing understanding of psychophysical relationships in man, has come into being. The integration of emotional factors with those of the somatic constitution has repercussions, both in the management of the individual patient and in the understanding of the immunological implications of why a particular individual fell sick with a particular malady at a particular time. This aspect of immunology is considered by Halliday (1948); it may be exemplified for upper respiratory infection by the recent monograph produced under the direction of H. G. Wolff of the New York Hospital (Holmes and others, 1950).

The present paper reports a study designed to show that personality factors play a part at least as important as other better-recognized factors in determining the resistance of individuals to various disease processes. It is the first limb of an investigation presenting a point of view in immunology which has been largely neglected in the past but may prove of fundamental importance to an understanding of disease 
as a reaction of the total organism to stresses requiring to be met by homeostatic adaptation, even though such an adaptation may be harmful and even fatal to the individual making it.

\section{EXPERIMENTAL METHOD}

A list of 110 disorders was compiled, 38 of which represented symptoms and complaints of a bodily nature and the remaining 72 actual somatic diseases and syndromes. The selection was made arbitrarily, those illnesses being included which were thought to represent a significant incidence in the population at risk. The disorders investigated are listed in Table I (pp. 52 to 57) where they are grouped by systems affected. Medical terminology was kept to a minimum, especially in the case of complaints, preference being given to popular lay terms which could be presumed to be understood by the majority of the subjects investigated. Fashions in the designation of aches and pains change with the times, and there is a considerable difference in local geography in this respect, different parts of the country having their own terms for certain disorders, but most of the terms used were readily comprehended by the subjects studied.

The list was prepared in questionnaire form, and instructions were added that the subject was to underline all those symptoms and illnesses from which he had ever suffered. He was assured that his answers would be treated confidentially, that his record would be used as in a census to represent an individual tally with respect to a total, and that no personal considerations would accrue from the pattern of his responses.

It was recognized at the outset that an investigation such as this could only approach validity providing sufficient healthy control subjects were used to give the results country-wide significance. The sampling methods employed and the techniques used in estimating reliability will be discussed later in this paper; let it suffice to say here that when copies of the questionnaire were filled in by the investigator after questioning a small batch of controls and were compared with those returned by mail from different parts of the country, no significant difference was found either in the absolute numbers of disorders recorded or in their differential pattern.

A consideration of the list of disorders will reveal no specifically gynaecological complaints or illnesses. For reasons dependent upon the sampling method employed, fewer females than males were available for study, and it was deemed advisable therefore to limit the disorders to those in which sex differences were not weighted in favour of female morbidity. In point of fact, little difference was found between the sexes for the disorders studied and for this reason no breakdown of the figures by sex is given.

\section{Method of Obtaining Data}

Apart from the small number of controls personally interviewed as already mentioned, the remaining healthy subjects were simply requested to fill out the questionnaire. This simple procedure did not, however, apply to most of the psychiatric patients. The neurotic group and those with character disorders were usually able to complete the questionnaire unaided, but for the psychotic groups personal interviews were used, each disorder being taken separately and the patient being asked if he had ever suffered from the disease or complaint in question. These answers, together with those of the other psychiatric groups included, were then checked by physical examination, interrogation of relatives, the medical history report of the hospital, and the records of other hospitals to which the patient had formerly been admitted. A final evaluation depended upon the sum of all the information garnered from these various sources. Surprisingly little extra information was however actually added by these time-consuming measures; except in the case of grossly deteriorated psychotics, the patients' self-assessments agreed well with the evidence supplied through such alternative channels. 
TABLE I

Percentage Incidence of 38 Bodily Complaints and 72 Organic Disorders

\begin{tabular}{|c|c|c|c|c|c|c|c|}
\hline \multirow{3}{*}{\multicolumn{2}{|c|}{$\begin{array}{c}\text { Tissue or Organ } \\
\text { System, etc., Involved }\end{array}$}} & \multirow{3}{*}{$\begin{array}{l}\text { Complaint or } \\
\text { Disorder }\end{array}$} & \multicolumn{5}{|c|}{ Percentage Incidence of Sorgatic } \\
\hline & & & \multirow[b]{2}{*}{ Controls } & \multirow[b]{2}{*}{$\begin{array}{l}\text { Psycho- } \\
\text { paths } \\
(42)\end{array}$} & \multicolumn{3}{|c|}{ Psycho-Neurosis } \\
\hline & & & & & $\begin{array}{c}\text { Anxiety } \\
\text { States } \\
\text { (61) }\end{array}$ & $\begin{array}{l}\text { Hysterics } \\
\quad(59)\end{array}$ & $\begin{array}{l}\omega \\
\text { Totat } \\
(120)\end{array}$ \\
\hline \multicolumn{2}{|c|}{$\begin{array}{l}\text { Infectious Fevers of } \\
\text { Childhood }\end{array}$} & $\begin{array}{l}\text { Measles } \\
\text { Rubella } \\
\text { Mumps } \\
\text { Chickenpox } \\
\text { Scarlatina } \\
\text { Diphtheria } \\
\text { Pertussis } \\
\text { Mastoiditis } \\
\text { Rheumatic fever }\end{array}$ & $\begin{array}{r}74 \cdot 9 \\
12 \cdot 4 \\
43 \cdot 2 \\
41 \cdot 5 \\
21 \cdot 5 \\
7 \cdot 1 \\
30 \cdot 5 \\
2 \cdot 0 \\
1 \cdot 7\end{array}$ & $\begin{array}{r}59 \cdot 5 \\
35 \cdot 0 \\
47 \cdot 6 \\
38 \cdot 1 \\
35 \cdot 7 \\
15 \cdot 0 \\
33 \cdot 3 \\
5 \cdot 0 \\
2 \cdot 4\end{array}$ & $\begin{array}{l}82 \cdot 0 \\
23 \cdot 5 \\
47 \cdot 5 \\
49 \cdot 2 \\
27 \cdot 9 \\
11 \cdot 8 \\
31 \cdot 1 \\
11 \cdot 8 \\
13 \cdot 1\end{array}$ & $\begin{array}{c}59 \cdot 3 \\
11 \cdot 1 \\
28 \cdot 8 \\
39 \cdot 0 \\
22 \cdot 0 \\
11 \cdot 1 \\
28 \cdot 8 \\
0 \\
10 \cdot 2\end{array}$ & $\begin{array}{l}70 \cdot \frac{80}{20} \\
19 \cdot \frac{2}{3} \\
38 \cdot 3 \\
44 \cdot 2 \\
25 \cdot 0 \\
11 \cdot 50 \\
30 \cdot 8 \\
7 \cdot \frac{8}{2} \\
11 \cdot 7\end{array}$ \\
\hline \multicolumn{2}{|c|}{ Congenital Defects, etc. } & $\begin{array}{l}\text { Flat feet } \\
\text { Fallen arches } \\
\text { Hernia } \\
\text { Undescended testis } \\
\text { Impacted wisdom teeth } \\
\text { Varicose veins } \\
\text { Infantile fits }\end{array}$ & $\begin{array}{l}8 \cdot 9 \\
4 \cdot 0 \\
7 \cdot 4 \\
1 \cdot 5 \\
0 \cdot 5 \\
4 \cdot 0 \\
1 \cdot 5\end{array}$ & $\begin{array}{l}4 \cdot 5 \\
0 \\
4 \cdot 5 \\
4 \cdot 5 \\
0 \\
9 \cdot 1 \\
0\end{array}$ & $\begin{array}{l}9 \cdot 1 \\
4 \cdot 5 \\
6 \cdot 8 \\
6 \cdot 8 \\
6 \cdot 8 \\
4 \cdot 5 \\
9 \cdot 1\end{array}$ & $\begin{array}{r}12 \cdot 0 \\
4 \cdot 0 \\
4 \cdot 0 \\
6 \cdot 0 \\
6 \cdot 0 \\
4 \cdot 0 \\
2 \cdot 0\end{array}$ & 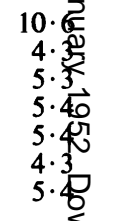 \\
\hline \multicolumn{2}{|c|}{$\begin{array}{l}\text { Other Childhood Complaints } \\
\text { and Disorders }\end{array}$} & $\begin{array}{l}\text { Sore throat } \\
\text { Tonsillectomy } \\
\text { Ear disease } \\
\text { Meningitis } \\
\text { Worm infestation } \\
\text { Rickets } \\
\text { Scurvy }\end{array}$ & $\begin{array}{l}70 \cdot 4 \\
30 \cdot 3 \\
6 \cdot 6 \\
1 \cdot 4 \\
4 \cdot 0 \\
0 \cdot 6 \\
0\end{array}$ & $\begin{array}{l}75 \cdot 0 \\
50 \cdot 0 \\
20 \cdot 0 \\
0 \\
9 \cdot 1 \\
0 \\
0\end{array}$ & $\begin{array}{r}82 \cdot 4 \\
29 \cdot 4 \\
47 \cdot 1 \\
6 \cdot 6 \\
4 \cdot 5 \\
4 \cdot 9 \\
2 \cdot 3\end{array}$ & $\begin{array}{c}100 \cdot 0 \\
33 \cdot 3 \\
0 \\
1 \cdot 6 \\
2 \cdot 0 \\
0 \\
0\end{array}$ & 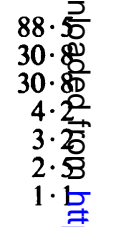 \\
\hline \multirow[t]{2}{*}{$\begin{array}{l}\text { Respiratory } \\
\text { System }\end{array}$} & Complaints & $\begin{array}{l}\text { Shortness of breath } \\
\text { Catarrh } \\
\text { Persistent cough } \\
\text { Haemoptysis }\end{array}$ & $\begin{array}{r}12 \cdot 4 \\
13 \cdot 8 \\
8 \cdot 8 \\
0 \cdot 7\end{array}$ & $\begin{array}{c}18 \cdot 2 \\
21 \cdot 4 \\
26 \cdot 2 \\
0\end{array}$ & $\begin{array}{r}36 \cdot 4 \\
27 \cdot 9 \\
19 \cdot 7 \\
5 \cdot 9\end{array}$ & $\begin{array}{c}32 \cdot 0 \\
13 \cdot 6 \\
13 \cdot 6 \\
0\end{array}$ & 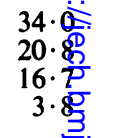 \\
\hline & Disorders & $\begin{array}{l}\text { Frequent colds } \\
\text { Feverish cold } \\
\text { Laryngitis } \\
\text { Bronchitis } \\
\text { Influenza } \\
\text { Pleurisy } \\
\text { Pneumonia } \\
\text { Tuberculosis } \\
\text { Bronchiectasis }\end{array}$ & $\begin{array}{r}52 \cdot 3 \\
14 \cdot 5 \\
11 \cdot 2 \\
8 \cdot 2 \\
52 \cdot 5 \\
2 \cdot 5 \\
10 \cdot 7 \\
1 \cdot 9 \\
0 \cdot 3\end{array}$ & $\begin{array}{r}71 \cdot 4 \\
20 \cdot 0 \\
10 \cdot 0 \\
19 \cdot 0 \\
38 \cdot 1 \\
5 \cdot 0 \\
14 \cdot 3 \\
2 \cdot 4 \\
2 \cdot 4\end{array}$ & $\begin{array}{l}55 \cdot 7 \\
23 \cdot 5 \\
17 \cdot 6 \\
19 \cdot 7 \\
67 \cdot 2 \\
17 \cdot 6 \\
18 \cdot 0 \\
1 \cdot 6 \\
0\end{array}$ & $\begin{array}{l}55 \cdot 9 \\
33 \cdot 3 \\
11 \cdot 1 \\
20 \cdot 3 \\
57 \cdot 6 \\
11 \cdot 1 \\
10 \cdot 2 \\
1 \cdot 7 \\
1 \cdot 7\end{array}$ & 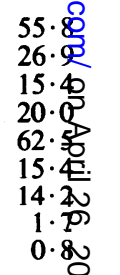 \\
\hline \multicolumn{2}{|l|}{ Allergy } & $\begin{array}{l}\text { Asthma } \\
\text { Hay fever } \\
\text { Eczema } \\
\text { Urticaria } \\
\text { Sensitive skin }\end{array}$ & $\begin{array}{r}1 \cdot 7 \\
4 \cdot 8 \\
11 \cdot 0 \\
3 \cdot 1 \\
5 \cdot 3\end{array}$ & $\begin{array}{l}0 \\
7 \cdot 1 \\
26 \cdot 2 \\
2 \cdot 4 \\
10 \cdot 0\end{array}$ & $\begin{array}{r}6 \cdot 6 \\
9 \cdot 8 \\
18 \cdot 0 \\
6 \cdot 6 \\
11 \cdot 8\end{array}$ & $\begin{array}{r}1 \cdot 7 \\
10 \cdot 2 \\
5 \cdot 1 \\
8 \cdot 5 \\
11 \cdot 1\end{array}$ & 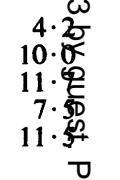 \\
\hline
\end{tabular}


The degree of significance was computed from $\chi^{2}$ tables in which the contributions of each of the psychiatric diagnostic groups (controls, psychopaths, neurotics, depressives and schizophrenics) could be assessed separately with respect to the total $\chi^{2}$ value (see Table II for example).

\begin{tabular}{|c|c|c|c|c|c|c|}
\hline \multicolumn{3}{|c|}{ Complaint or Disorder } & \multirow{3}{*}{$\chi^{2}$} & \multirow{3}{*}{$\begin{array}{c}\text { Confidence Level } \\
\text { of Probability } \\
\boldsymbol{P} \\
\text { Per cent. }\end{array}$} & \multirow{3}{*}{$\begin{array}{l}\text { Principal } \\
\text { Psychiatric } \\
\text { Disorder } \\
\text { contributing } \\
\text { to this } \chi^{2}\end{array}$} & \multirow{3}{*}{$\begin{array}{r}\text { Extent of } \\
\text { Contribution } \\
\text { to Total } \chi^{2}\end{array}$} \\
\hline \multicolumn{3}{|c|}{ Psychosis } & & & & \\
\hline $\begin{array}{l}\text { Depres- } \\
\text { sives } \\
(59)\end{array}$ & $\begin{array}{l}\text { Schizo- } \\
\text { phrenics } \\
\text { (51) }\end{array}$ & $\begin{array}{l}\text { Total } \\
(110)\end{array}$ & & & & \\
\hline $\begin{array}{r}79 \cdot 7 \\
28 \cdot 8 \\
44 \cdot 1 \\
54 \cdot 2 \\
22 \cdot 0 \\
16 \cdot 9 \\
55 \cdot 9 \\
3 \cdot 4 \\
6 \cdot 8\end{array}$ & $\begin{array}{r}86 \cdot 3 \\
29 \cdot 4 \\
35 \cdot 3 \\
52 \cdot 9 \\
27 \cdot 5 \\
19 \cdot 6 \\
56 \cdot 9 \\
2 \cdot 0 \\
7 \cdot 8\end{array}$ & $\begin{array}{r}82 \cdot 7 \\
29 \cdot 1 \\
40 \cdot 0 \\
53 \cdot 6 \\
24 \cdot 5 \\
18 \cdot 2 \\
56 \cdot 4 \\
2 \cdot 7 \\
7 \cdot 3\end{array}$ & $\begin{array}{r}4 \cdot 6 \\
21 \cdot 1 \\
1 \cdot 2 \\
1 \cdot 9 \\
4 \cdot 6 \\
12 \cdot 6 \\
17 \cdot 7 \\
3 \cdot 0 \\
22 \cdot 0\end{array}$ & $\begin{array}{l}\text { N.S. } \\
0 \cdot 1 \\
\text { N.S. } \\
\text { N.S. } \\
\text { N.S. } \\
2 \cdot 5 \\
0 \cdot 5 \\
\text { N.S. } \\
0 \cdot 1\end{array}$ & $\begin{array}{l}\qquad \overline{\text { All }} \\
- \\
- \\
\text { Psychosis } \\
\text { Psychosis } \\
\text { Neurosis }\end{array}$ & $\begin{array}{l}- \\
- \\
- \\
\overline{8} \cdot 4 \\
14 \cdot 8 \\
\overline{13 \cdot 1}\end{array}$ \\
\hline $\begin{array}{l}- \\
- \\
- \\
-\end{array}$ & $\begin{array}{l}- \\
- \\
- \\
-\end{array}$ & $\begin{array}{l}- \\
- \\
- \\
-\end{array}$ & $\begin{array}{r}0 \cdot 8 \\
0 \cdot 02 \\
0 \cdot 6 \\
5 \cdot 2 \\
10 \cdot 1 \\
1 \cdot 2 \\
4 \cdot 6\end{array}$ & $\begin{array}{l}\text { N.S. } \\
\text { N.S. } \\
\text { N.S. } \\
\text { N.S. } \\
1 \cdot 0 \\
\text { N.S. } \\
5 \cdot 0\end{array}$ & $\begin{array}{r}\frac{Z}{-} \\
\text { Neurosis } \\
\text { Neurosis }\end{array}$ & $\begin{array}{l}- \\
\overline{-} \\
\overline{7 \cdot 0} \\
\overline{2 \cdot 8}\end{array}$ \\
\hline $\begin{array}{r}91 \cdot 5 \\
35 \cdot 6 \\
13 \cdot 6 \\
3 \cdot 4 \\
\overline{6 \cdot 8} \\
-\end{array}$ & $\begin{array}{l}96 \cdot 1 \\
37 \cdot 3 \\
23 \cdot 5 \\
0 \\
\overline{5 \cdot 9} \\
-\end{array}$ & $\begin{array}{l}93 \cdot 6 \\
36 \cdot 4 \\
18 \cdot 2 \\
1 \cdot 8 \\
\overline{6 \cdot 4} \\
-\end{array}$ & $\begin{array}{r}22 \cdot 0 \\
3 \cdot 6 \\
17 \cdot 8 \\
7 \cdot 3 \\
1 \cdot 6 \\
16 \cdot 0 \\
-\end{array}$ & $\begin{array}{l}0.1 \\
\text { N.S. } \\
0.5 \\
\text { N.S. } \\
\text { N.S. } \\
0.5 \\
\text { N.S. }\end{array}$ & $\begin{array}{l}\text { Schizophrenia } \\
\text { Neurosis } \\
- \\
\text { - } \\
\text { Depression }\end{array}$ & $\begin{array}{l}7 \cdot 7 \\
\overline{6 \cdot 5} \\
\overline{-} \\
\overline{7 \cdot 4}\end{array}$ \\
\hline $\begin{array}{r}-19 \cdot 0 \\
25 \cdot 4 \\
3 \cdot 4\end{array}$ & $\begin{array}{r}31 \cdot 4 \\
29 \cdot 4 \\
9 \cdot 8\end{array}$ & $\begin{array}{r}35 \cdot 5 \\
27 \cdot 3 \\
6 \cdot 4\end{array}$ & $\begin{array}{l}15 \cdot 8 \\
21 \cdot 3 \\
23 \cdot 6 \\
11 \cdot 7\end{array}$ & $\begin{array}{l}0 \cdot 1 \\
0 \cdot 1 \\
0 \cdot 1 \\
2 \cdot 5\end{array}$ & $\begin{array}{l}\text { Neurosis } \\
\text { Depression } \\
\text { Schizophrenia } \\
\text { Schizophrenia }\end{array}$ & $\begin{array}{r}11 \cdot 0 \\
11 \cdot 6 \\
7 \cdot 7 \\
8 \cdot 4\end{array}$ \\
\hline $\begin{array}{l}71 \cdot 2 \\
28 \cdot 8 \\
20 \cdot 3 \\
15 \cdot 3 \\
98 \cdot 3 \\
11 \cdot 9 \\
18 \cdot 6 \\
6 \cdot 8 \\
0\end{array}$ & $\begin{array}{r}78 \cdot 5 \\
54 \cdot 9 \\
9 \cdot 8 \\
19 \cdot 6 \\
80 \cdot 4 \\
9 \cdot 8 \\
21 \cdot 6 \\
3 \cdot 9 \\
2 \cdot 0\end{array}$ & $\begin{array}{r}74 \cdot 5 \\
40 \cdot 9 \\
15 \cdot 5 \\
17 \cdot 3 \\
90 \cdot 0 \\
10 \cdot 9 \\
20 \cdot 0 \\
5 \cdot 5 \\
0.9\end{array}$ & $\begin{array}{r}8 \cdot 6 \\
33 \cdot 8 \\
4 \cdot 0 \\
14 \cdot 2 \\
40 \cdot 9 \\
\overline{5 \cdot} \\
5 \cdot 8 \\
4 \cdot 9\end{array}$ & $\begin{array}{l}\text { N.S. } \\
0 \cdot 1 \\
\text { N.S. } \\
1 \cdot 0 \\
0 \cdot 1 \\
\frac{1}{\text { N.S. }} \\
\text { N.S. } \\
\text { N.S. }\end{array}$ & $\begin{array}{l}\text { Schizophrenia } \\
\text { Neurosis } \\
\text { Depression } \\
\quad- \\
\quad- \\
-\end{array}$ & $\begin{array}{l}\overline{23 \cdot 7} \\
\overline{5 \cdot 0} \\
14 \cdot 4 \\
- \\
- \\
-\end{array}$ \\
\hline $\begin{array}{l}1 \cdot 7 \\
13 \cdot 6 \\
50 \cdot 8 \\
10 \cdot 2 \\
27 \cdot 1\end{array}$ & $\begin{array}{r}3 \cdot 9 \\
19 \cdot 6 \\
45 \cdot 1 \\
11 \cdot 8 \\
33 \cdot 3\end{array}$ & $\begin{array}{r}2 \cdot 7 \\
16 \cdot 4 \\
48 \cdot 2 \\
10 \cdot 9 \\
30 \cdot 0\end{array}$ & $\begin{array}{r}4 \cdot 1 \\
13 \cdot 6 \\
67 \cdot 7 \\
11 \cdot 8 \\
27 \cdot 6\end{array}$ & $\begin{array}{l}\text { N.S. } \\
1 \cdot 0 \\
0 \cdot 1 \\
2 \cdot 5 \\
0 \cdot 1\end{array}$ & $\begin{array}{l}\text { Schizophrenia } \\
\text { Depression } \\
\text { Schizophrenia } \\
\text { Schizophrenia }\end{array}$ & $\begin{array}{r}\overline{6 \cdot 1} \\
33 \cdot 2 \\
4 \cdot 2 \\
11 \cdot 7\end{array}$ \\
\hline
\end{tabular}


TABLE I-cont.

\begin{tabular}{|c|c|c|c|c|c|c|c|}
\hline \multirow{3}{*}{\multicolumn{2}{|c|}{$\begin{array}{c}\text { Tissue or Organ } \\
\text { System, etc., Involved }\end{array}$}} & \multirow{3}{*}{$\begin{array}{l}\text { Complaint or } \\
\text { Disorder }\end{array}$} & \multirow[b]{3}{*}{$\begin{array}{c}\text { Controls } \\
\text { (354) }\end{array}$} & \multirow[b]{3}{*}{$\begin{array}{l}\text { Psycho- } \\
\text { paths } \\
(42)\end{array}$} & \multicolumn{3}{|c|}{ Percentage Incidence of Sophatic } \\
\hline & & & & & \multicolumn{3}{|c|}{ Psycho-Neurosis } \\
\hline & & & & & $\begin{array}{l}\text { Anxiety } \\
\text { States } \\
(61)\end{array}$ & $\begin{array}{l}\text { Hysterics } \\
\text { (59) }\end{array}$ & $\begin{array}{l}\text { Totåำ } \\
\text { (12ब) }\end{array}$ \\
\hline \multirow[t]{2}{*}{$\begin{array}{l}\text { Gastro-Intestinal } \\
\text { Tract }\end{array}$} & Complaints & $\begin{array}{l}\text { Constipation } \\
\text { Diarrhoea } \\
\text { Stomach troubles } \\
\text { Indigestion } \\
\text { Gastric stomach } \\
\text { Pain after meals } \\
\text { Nausea and vomiting } \\
\text { Bilious attacks } \\
\text { Vomiting blood } \\
\text { Blood in stools } \\
\text { Colic } \\
\text { Jaundice }\end{array}$ & $\begin{array}{r}20 \cdot 6 \\
29 \cdot 1 \\
7 \cdot 6 \\
20 \cdot 4 \\
2 \cdot 6 \\
3 \cdot 9 \\
17 \cdot 8 \\
13 \cdot 6 \\
0 \cdot 7 \\
0 \\
4 \cdot 6 \\
5 \cdot 9\end{array}$ & $\begin{array}{l}23 \cdot 8 \\
23 \cdot 8 \\
14 \cdot 3 \\
20 \cdot 0 \\
5 \cdot 0 \\
10 \cdot 0 \\
30 \cdot 0 \\
19 \cdot 0 \\
0 \\
5 \cdot 0 \\
0 \\
0\end{array}$ & $\begin{array}{l}41 \cdot 0 \\
44 \cdot 3 \\
24 \cdot 6 \\
52 \cdot 9 \\
0 \\
41 \cdot 2 \\
52 \cdot 9 \\
27 \cdot 9 \\
5 \cdot 9 \\
11 \cdot 8 \\
5 \cdot 9 \\
17 \cdot 6\end{array}$ & $\begin{array}{l}27 \cdot 1 \\
18 \cdot 6 \\
22 \cdot 0 \\
77 \cdot 8 \\
22 \cdot 2 \\
22 \cdot 2 \\
55 \cdot 6 \\
27 \cdot 1 \\
0 \\
22 \cdot 2 \\
0 \\
11 \cdot 1\end{array}$ & 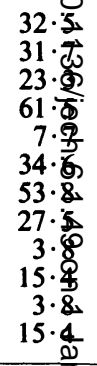 \\
\hline & Disorders & $\begin{array}{l}\text { Appendicitis } \\
\text { Chronic appendix } \\
\text { Appendectomy } \\
\text { Peritonitis } \\
\text { Peptic ulcer } \\
\text { Piles } \\
\text { Dysentery } \\
\text { Gallstones } \\
\text { Infective hepatitis }\end{array}$ & $\begin{array}{r}9 \cdot 3 \\
3 \cdot 9 \\
13 \cdot 8 \\
1 \cdot 3 \\
1 \cdot 7 \\
9 \cdot 0 \\
5 \cdot 4 \\
0 \cdot 3 \\
2 \cdot 6\end{array}$ & $\begin{array}{l}10 \cdot 0 \\
45 \cdot 0 \\
20 \cdot 0 \\
5 \cdot 0 \\
0 \\
9 \cdot 5 \\
25 \cdot 0 \\
0 \\
0\end{array}$ & $\begin{array}{l}11 \cdot 8 \\
24 \cdot 4 \\
5 \cdot 9 \\
0 \\
2 \cdot 3 \\
16 \cdot 4 \\
11 \cdot 8 \\
0 \\
5 \cdot 9\end{array}$ & $\begin{array}{l}11 \cdot 1 \\
33 \cdot 3 \\
11 \cdot 1 \\
0 \\
0 \\
1 \cdot 7 \\
11 \cdot 1 \\
0 \\
0\end{array}$ & 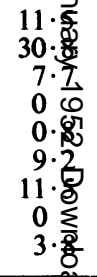 \\
\hline \multirow[t]{2}{*}{$\begin{array}{l}\text { Central Nervous } \\
\text { System }\end{array}$} & Complaints & $\begin{array}{l}\text { Headache } \\
\text { Recurrent headache } \\
\text { Vertigo } \\
\text { Blurred vision } \\
\text { Syncope } \\
\text { "Turns" } \\
\text { "Absences" } \\
\text { Déjà vu } \\
\text { Depersonalization } \\
\text { "Watched self act" } \\
\text { Collapse }\end{array}$ & $\begin{array}{r}32 \cdot 8 \\
7 \cdot 2 \\
5 \cdot 3 \\
7 \cdot 2 \\
12 \cdot 4 \\
0 \cdot 8 \\
1 \cdot 5 \\
10 \cdot 4 \\
0 \cdot 5 \\
0 \cdot 5 \\
0\end{array}$ & $\begin{array}{l}31 \cdot 0 \\
25 \cdot 0 \\
35 \cdot 0 \\
30 \cdot 0 \\
38 \cdot 1 \\
20 \cdot 0 \\
0 \\
22 \cdot 7 \\
4 \cdot 5 \\
0 \\
0\end{array}$ & $\begin{array}{r}47 \cdot 5 \\
35 \cdot 3 \\
41 \cdot 2 \\
35 \cdot 3 \\
42 \cdot 6 \\
17 \cdot 6 \\
4 \cdot 5 \\
22 \cdot 7 \\
2 \cdot 3 \\
2 \cdot 3 \\
9 \cdot 1\end{array}$ & $\begin{array}{l}35 \cdot 6 \\
55 \cdot 6 \\
33 \cdot 3 \\
22 \cdot 2 \\
59 \cdot 3 \\
0 \\
0 \\
14 \cdot 0 \\
2 \cdot 0 \\
0 \\
12 \cdot 0\end{array}$ & 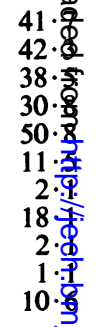 \\
\hline & Disorders & $\begin{array}{l}\text { Head injury } \\
\text { Accident } \\
\text { Heat stroke } \\
\text { Herpes zoster } \\
\text { Neuritis } \\
\text { Migraine } \\
\text { Fits (epilepsy) }\end{array}$ & $\begin{array}{l}24 \cdot 3 \\
23 \cdot 3 \\
1 \cdot 3 \\
0 \cdot 8 \\
7 \cdot 6 \\
8 \cdot 2 \\
0 \cdot 8\end{array}$ & $\begin{array}{c}27 \cdot 5 \\
27 \cdot 3 \\
5 \cdot 0 \\
0 \\
7 \cdot 1 \\
11 \cdot 9 \\
4 \cdot 8\end{array}$ & $\begin{array}{r}54 \cdot 5 \\
25 \cdot 0 \\
17 \cdot 6 \\
1 \cdot 6 \\
26 \cdot 2 \\
37 \cdot 7 \\
4 \cdot 9\end{array}$ & $\begin{array}{r}40 \cdot 0 \\
40 \cdot 0 \\
11 \cdot 1 \\
1 \cdot 7 \\
15 \cdot 2 \\
25 \cdot 4 \\
6 \cdot 8\end{array}$ & $\begin{array}{r}46 \cdot 8 \\
33 \cdot 8 \\
15 \cdot 8 \\
1 \cdot 3 \\
20 \cdot 8 \\
31 \cdot \frac{8}{4} \\
5 \cdot \frac{8}{8.8}\end{array}$ \\
\hline \multirow[t]{2}{*}{$\begin{array}{l}\text { Cardiovascular } \\
\text { System }\end{array}$} & Complaints & $\begin{array}{l}\text { Palpitation } \\
\text { Epistaxis } \\
\text { Swollen ankles }\end{array}$ & $\begin{array}{r}5 \cdot 4 \\
21 \cdot 1 \\
3 \cdot 5\end{array}$ & $\begin{array}{r}0 \\
25 \cdot 0 \\
4 \cdot 5\end{array}$ & $\begin{array}{r}13 \cdot 6 \\
52 \cdot 9 \\
6 \cdot 9\end{array}$ & $\begin{array}{r}14 \cdot 0 \\
22 \cdot 2 \\
2 \cdot 0\end{array}$ & 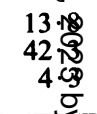 \\
\hline & Disorders & $\begin{array}{l}\text { Heart trouble } \\
\text { Blood pressure } \\
\text { Anaemia }\end{array}$ & $\begin{array}{l}1 \cdot 1 \\
1 \cdot 1 \\
2 \cdot 3\end{array}$ & $\begin{array}{l}2 \cdot 4 \\
0 \\
4 \cdot 8\end{array}$ & $\begin{array}{l}4 \cdot 9 \\
4 \cdot 9 \\
1 \cdot 6\end{array}$ & $\begin{array}{l}3 \cdot 4 \\
5 \cdot 1 \\
3 \cdot 4\end{array}$ & $\begin{array}{l}4 \frac{9}{5} \\
5 \frac{0}{9} \\
2: 3\end{array}$ \\
\hline & & & & & & & 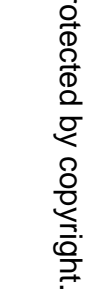 \\
\hline
\end{tabular}




\begin{tabular}{|c|c|c|c|c|c|c|}
\hline \multicolumn{3}{|c|}{ Complaint or Disorder } & \multirow{3}{*}{$\chi^{2}$} & \multirow{3}{*}{$\begin{array}{c}\text { Confidence Level } \\
\text { of Probability } \\
\boldsymbol{P} \\
\text { Per cent. }\end{array}$} & \multirow{3}{*}{$\begin{array}{l}\text { Principal } \\
\text { Psychiatric } \\
\text { Disorder } \\
\text { contributing } \\
\text { to this } \chi^{2}\end{array}$} & \multirow{3}{*}{$\begin{array}{c}\text { Extent of } \\
\text { Contribution } \\
\text { to Total } \chi^{2}\end{array}$} \\
\hline \multicolumn{3}{|c|}{ Psychosis } & & & & \\
\hline $\begin{array}{c}\text { Depres- } \\
\text { sives } \\
(59)\end{array}$ & $\begin{array}{l}\text { Schizo- } \\
\text { phrenics } \\
\text { (51) }\end{array}$ & $\begin{array}{l}\text { Total } \\
\text { (110) }\end{array}$ & & & & \\
\hline $\begin{aligned} 79 \cdot 7 \\
54 \cdot 2 \\
49 \cdot 1 \\
74 \cdot 6 \\
30 \cdot 5 \\
37 \cdot 3 \\
66 \cdot 1 \\
39 \cdot 0 \\
0 \\
6 \cdot 8 \\
15 \cdot 3 \\
8 \cdot 5\end{aligned}$ & $\begin{array}{r}74 \cdot 5 \\
62 \cdot 7 \\
37 \cdot 3 \\
68 \cdot 6 \\
11 \cdot 8 \\
51 \cdot 0 \\
74 \cdot 5 \\
66 \cdot 7 \\
5 \cdot 9 \\
17 \cdot 6 \\
9 \cdot 8 \\
13 \cdot 7\end{array}$ & $\begin{array}{r}77 \cdot 3 \\
58 \cdot 2 \\
43 \cdot 6 \\
71 \cdot 8 \\
21 \cdot 8 \\
43 \cdot 6 \\
70 \cdot 0 \\
41 \cdot 8 \\
2 \cdot 7 \\
11 \cdot 8 \\
12 \cdot 7 \\
10 \cdot 9\end{array}$ & $\begin{array}{r}85 \cdot 9 \\
23 \cdot 5 \\
67 \cdot 8 \\
49 \cdot 8 \\
27 \cdot 7 \\
53 \cdot 5 \\
46 \cdot 5 \\
63 \cdot 1 \\
8 \cdot 7 \\
24 \cdot 1 \\
9 \cdot 2 \\
6 \cdot 4\end{array}$ & $\begin{array}{l}0.1 \\
0.1 \\
0 \cdot 1 \\
0 \cdot 1 \\
0 \cdot 1 \\
0 \cdot 1 \\
0 \cdot 1 \\
0 \cdot 1 \\
\text { N.S. } \\
0.1 \\
\text { N.S. } \\
\text { N.S. }\end{array}$ & \begin{tabular}{l} 
Depression \\
Psychosis \\
Depression \\
Psychosis \\
Depression \\
Psychosis \\
Psychosis \\
Schizophrenia \\
Schizophrenia \\
\multicolumn{1}{c}{ - }
\end{tabular} & $\begin{array}{l}41 \cdot 4 \\
19 \cdot 1 \\
34 \cdot 2 \\
28 \cdot 0 \\
12 \cdot 1 \\
28 \cdot 9 \\
24 \cdot 0 \\
41 \cdot 2 \\
\overline{15 \cdot 7} \\
=\end{array}$ \\
\hline $\begin{array}{r}13 \cdot 6 \\
44 \cdot 1 \\
10 \cdot 2 \\
1 \cdot 7 \\
8 \cdot 5 \\
39 \cdot 0 \\
18 \cdot 6 \\
3 \cdot 4 \\
5 \cdot 1\end{array}$ & $\begin{array}{r}13 \cdot 7 \\
35 \cdot 3 \\
13 \cdot 7 \\
3 \cdot 9 \\
1 \cdot 9 \\
23 \cdot 5 \\
9 \cdot 8 \\
0 \\
3 \cdot 9\end{array}$ & $\begin{array}{r}13 \cdot 6 \\
40 \cdot 0 \\
11 \cdot 8 \\
2.7 \\
5 \cdot 5 \\
31 \cdot 8 \\
14 \cdot 5 \\
1.8 \\
4 \cdot 5\end{array}$ & $\begin{array}{r}3 \cdot 5 \\
58 \cdot 7 \\
1 \cdot 7 \\
2 \cdot 9 \\
13 \cdot 5 \\
39 \cdot 9 \\
21 \cdot 0 \\
\overline{1.6}\end{array}$ & $\begin{array}{l}\text { N.S. } \\
0 \cdot 1 \\
\text { N.S. } \\
\text { N.S. } \\
1.0 \\
0 \cdot 1 \\
0 \cdot 1 \\
\text { N.S. } \\
\text { N.S. }\end{array}$ & $\begin{array}{c}- \\
\text { Depression } \\
- \\
\text { Depression } \\
\text { Depression } \\
\text { Depression } \\
- \\
-\end{array}$ & $\begin{array}{c}\overline{17 \cdot 4} \\
\overline{-} \\
12 \cdot 4 \\
30 \cdot 6 \\
8 \cdot 2 \\
=\end{array}$ \\
\hline $\begin{array}{l}74 \cdot 6 \\
55 \cdot 9 \\
39 \cdot 0 \\
40 \cdot 7 \\
40 \cdot 7 \\
15 \cdot 3 \\
= \\
= \\
- \\
-\end{array}$ & $\begin{array}{l}96 \cdot 1 \\
43 \cdot 1 \\
49 \cdot 0 \\
43 \cdot 1 \\
54 \cdot 9 \\
31 \cdot 4 \\
= \\
= \\
= \\
-\end{array}$ & $\begin{array}{l}84 \cdot 5 \\
50 \cdot 0 \\
43 \cdot 6 \\
41 \cdot 8 \\
47 \cdot 3 \\
22 \cdot 7 \\
= \\
= \\
= \\
-\end{array}$ & $\begin{array}{l}55 \cdot 8 \\
63 \cdot 8 \\
45 \cdot 5 \\
34 \cdot 5 \\
75 \cdot 1 \\
41 \cdot 4 \\
- \\
13 \cdot 4 \\
3 \cdot 6 \\
0 \cdot 5 \\
-\end{array}$ & $\begin{array}{l}0 \cdot 1 \\
0 \cdot 1 \\
0 \cdot 1 \\
0 \cdot 1 \\
0 \cdot 1 \\
0 \cdot 1 \\
\text { N.S. } \\
0 \cdot 5 \\
\text { N.S. } \\
\text { N.S. } \\
\text { N.S. }\end{array}$ & $\begin{array}{l}\text { Schizophrenia } \\
\text { Depression } \\
\text { Schizophrenia } \\
\text { Psychosis } \\
\text { Neurosis } \\
\text { Schizophrenia } \\
\text { Neurosis } \\
\text { - } \\
\text { - }\end{array}$ & $\begin{array}{l}32 \cdot 6 \\
26 \cdot 1 \\
13 \cdot 8 \\
17 \cdot 0 \\
26 \cdot 3 \\
22 \cdot 8 \\
- \\
8 \cdot 1 \\
= \\
=\end{array}$ \\
\hline $\begin{array}{r}- \\
\overline{15 \cdot 3} \\
10 \cdot 2 \\
44 \cdot 1 \\
22 \cdot 0 \\
3 \cdot 4\end{array}$ & $\begin{array}{l}- \\
\overline{7} \\
9 \cdot 8 \\
5 \cdot 9 \\
43 \cdot 1 \\
13 \cdot 7 \\
15 \cdot 6\end{array}$ & $\begin{array}{r}- \\
1 \overline{12 \cdot 7} \\
8 \cdot 2 \\
43 \cdot 6 \\
18 \cdot 2 \\
9 \cdot 1\end{array}$ & $\begin{array}{r}10 \cdot 7 \\
2 \cdot 5 \\
25 \cdot 5 \\
70 \cdot 0 \\
35 \cdot 8 \\
30 \cdot 1\end{array}$ & $\begin{array}{l}0.5 \\
\text { N.S. } \\
0 \cdot 1 \\
0 \cdot 1 \\
0 \cdot 1 \\
0 \cdot 1 \\
0 \cdot 1\end{array}$ & \begin{tabular}{l} 
Neurosis \\
\multicolumn{1}{|}{-} \\
Depression \\
Psychosis \\
Neurosis \\
Schizophrenia
\end{tabular} & $\begin{array}{l}7 \cdot 6 \\
\overline{-} \\
18 \cdot 5 \\
50 \cdot 0 \\
23 \cdot 2 \\
21 \cdot 0\end{array}$ \\
\hline $2 \overline{28 \cdot 8}$ & $\begin{array}{l}\overline{62 \cdot 7} \\
-\end{array}$ & $\begin{array}{l}\overline{44 \cdot 5} \\
-\end{array}$ & $\begin{array}{r}7 \cdot 8 \\
22 \cdot 6 \\
1 \cdot 5\end{array}$ & $\begin{array}{l}2 \cdot 5 \\
0 \cdot 1 \\
\text { N.S. }\end{array}$ & $\begin{array}{l}\text { Neurosis } \\
\text { Schizophrenia } \\
\text { - }\end{array}$ & $\begin{array}{r}5 \cdot 0 \\
16 \cdot 0 \\
-\end{array}$ \\
\hline $\begin{array}{r}3 \cdot 4 \\
13 \cdot 6 \\
22 \cdot 0\end{array}$ & $\begin{array}{r}9 \cdot 8 \\
19 \cdot 6 \\
25 \cdot 5\end{array}$ & $\begin{array}{r}6 \cdot 4 \\
16 \cdot 4 \\
23 \cdot 6\end{array}$ & $\begin{array}{l}13 \cdot 8 \\
46 \cdot 4 \\
66 \cdot 0\end{array}$ & $\begin{array}{l}1 \cdot 0 \\
0 \cdot 1 \\
0 \cdot 1\end{array}$ & $\begin{array}{l}\text { Schizophrenia } \\
\text { Schizophrenia } \\
\text { Schizophrenia }\end{array}$ & $\begin{array}{r}9 \cdot 5 \\
24 \cdot 6 \\
30 \cdot 6\end{array}$ \\
\hline
\end{tabular}


TABLE I-cont.

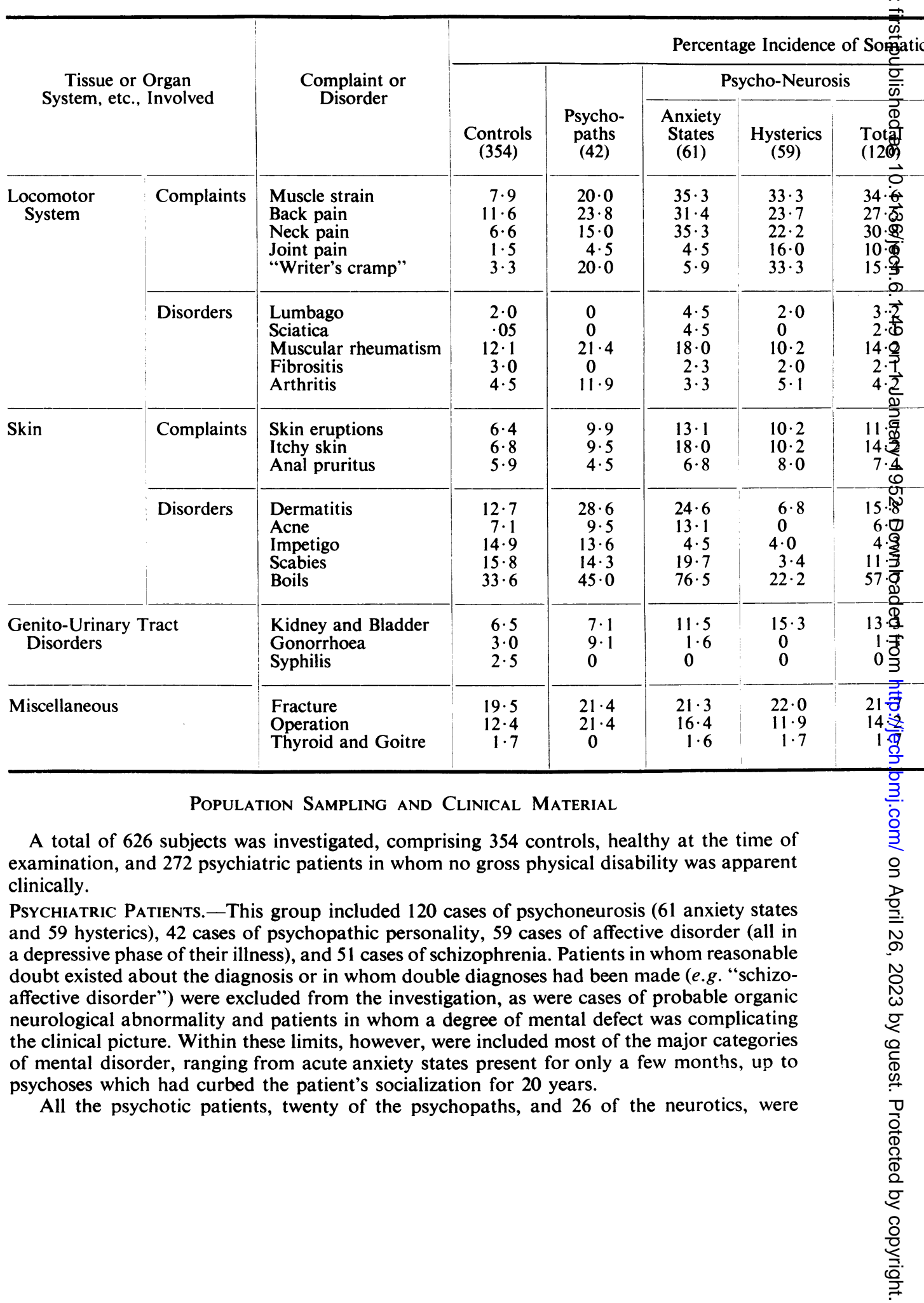




\begin{tabular}{|c|c|c|c|c|c|c|}
\hline \multicolumn{3}{|c|}{ Complaint or Disorder } & \multirow{3}{*}{$\chi^{2}$} & \multirow{3}{*}{$\begin{array}{c}\text { Confidence Level } \\
\text { of Probability } \\
P \\
\text { Per cent. }\end{array}$} & \multirow{3}{*}{$\begin{array}{l}\text { Principal } \\
\text { Psychiatric } \\
\text { Disorder } \\
\text { contributing } \\
\text { to this } \chi^{2}\end{array}$} & \multirow{3}{*}{$\begin{array}{c}\text { Extent of } \\
\text { Contribution } \\
\text { to Total } \chi^{2}\end{array}$} \\
\hline \multicolumn{3}{|c|}{ Psychosis } & & & & \\
\hline $\begin{array}{l}\text { Depres- } \\
\text { sives } \\
(59)\end{array}$ & $\begin{array}{l}\text { Schizo- } \\
\text { phrenics } \\
\quad(51)\end{array}$ & $\begin{array}{l}\text { Total } \\
(110)\end{array}$ & & & & \\
\hline $\begin{array}{c}15 \cdot 3 \\
42 \cdot 4 \\
20 \cdot 3 \\
-\frac{6}{6 \cdot 8}\end{array}$ & $\begin{array}{l}39 \cdot 2 \\
52 \cdot 9 \\
45 \cdot 1 \\
-\overline{31 \cdot 4}\end{array}$ & $\begin{array}{c}26 \cdot 4 \\
47 \cdot 3 \\
31 \cdot 8 \\
\overline{18 \cdot 2}\end{array}$ & $\begin{array}{l}29 \cdot 2 \\
53 \cdot 2 \\
24 \cdot 2 \\
12 \cdot 2 \\
30 \cdot 6\end{array}$ & $\begin{array}{l}0 \cdot 1 \\
0 \cdot 1 \\
0 \cdot 1 \\
0 \cdot 5 \\
0 \cdot 1\end{array}$ & $\begin{array}{l}\text { Schizophrenia } \\
\text { Schizophrenia } \\
\text { Schizophrenia } \\
\text { Neurosis } \\
\text { Schizophrenia }\end{array}$ & $\begin{array}{r}18 \cdot 2 \\
22 \cdot 9 \\
20 \cdot 5 \\
8 \cdot 3 \\
20 \cdot 3\end{array}$ \\
\hline $\begin{array}{l}\overline{-} \\
\overline{35 \cdot 6} \\
\overline{27 \cdot 1}\end{array}$ & $\begin{array}{l}\overline{-} \\
\overline{56 \cdot 9} \\
\overline{23 \cdot 5}\end{array}$ & $\begin{array}{l}\overline{-} \\
\overline{45 \cdot 5} \\
\overline{25 \cdot 5}\end{array}$ & $\begin{array}{r}0 \cdot 9 \\
2 \cdot 2 \\
57 \cdot 4 \\
0 \cdot 8 \\
46 \cdot 5\end{array}$ & $\begin{array}{l}\text { N.S. } \\
\text { N.S. } \\
0 \cdot 1 \\
\text { N.S. } \\
0 \cdot 1\end{array}$ & $\begin{array}{l}-\overline{-} \\
\text { Schizophrenia } \\
\text { Psychosis }\end{array}$ & $\begin{array}{l}\overline{-} \\
\overline{38 \cdot 5} \\
\overline{36 \cdot 8}\end{array}$ \\
\hline $\begin{array}{r}8 \cdot 5 \\
33 \cdot 9 \\
-\end{array}$ & $\begin{array}{c}17 \cdot 6 \\
35 \cdot 3 \\
-\end{array}$ & $\begin{array}{c}12 \cdot 7 \\
34 \cdot 5 \\
-\end{array}$ & $\begin{array}{r}25 \cdot 2 \\
49 \cdot 6 \\
0 \cdot 3\end{array}$ & $\begin{array}{l}0 \cdot 1 \\
0 \cdot 1 \\
\text { N.S. }\end{array}$ & $\begin{array}{l}\text { Schizophrenia } \\
\text { Psychosis } \\
\text { _ }\end{array}$ & $\begin{array}{c}11 \cdot 1 \\
38 \cdot 0 \\
-\end{array}$ \\
\hline $\begin{array}{l}50 \cdot 8 \\
28 \cdot 8 \\
\overline{15 \cdot 3} \\
54 \cdot 2\end{array}$ & $\begin{array}{l}45 \cdot 1 \\
23 \cdot 5 \\
- \\
21 \cdot 6 \\
64 \cdot 7\end{array}$ & $\begin{array}{l}48 \cdot 2 \\
26 \cdot 4 \\
- \\
18 \cdot 2 \\
59 \cdot 1\end{array}$ & $\begin{array}{r}54 \cdot 7 \\
15 \cdot 6 \\
6 \cdot 9 \\
2 \cdot 4 \\
10 \cdot 8\end{array}$ & $\begin{array}{l}0 \cdot 1 \\
0 \cdot 5 \\
5 \cdot 0 \\
\text { N.S. } \\
5 \cdot 0\end{array}$ & $\begin{array}{l}\text { Depression } \\
\text { Schizophrenia } \\
\text { Controls } \\
\text { Schizophrenia }\end{array}$ & $\begin{array}{r}26 \cdot 2 \\
8 \cdot 2 \\
4 \cdot 3 \\
\overline{4 \cdot 2}\end{array}$ \\
\hline $\begin{array}{c}23 \cdot 7 \\
0 \\
0\end{array}$ & $\begin{array}{c}31 \cdot 4 \\
0 \\
0\end{array}$ & $\begin{array}{c}27 \cdot 3 \\
0 \\
0\end{array}$ & $\begin{array}{r}33 \cdot 9 \\
4 \cdot 1 \\
-\end{array}$ & $\begin{array}{l}0 \cdot 1 \\
\text { N.S. } \\
\text { N.S. }\end{array}$ & $\begin{array}{c}\text { Schizophrenia } \\
- \\
-\end{array}$ & $\begin{array}{c}17 \cdot 5 \\
- \\
-\end{array}$ \\
\hline $\begin{array}{r}23 \cdot 7 \\
18 \cdot 6 \\
1 \cdot 7\end{array}$ & $\begin{array}{r}27 \cdot 5 \\
33 \cdot 3 \\
3 \cdot 9\end{array}$ & $\begin{array}{r}25 \cdot 5 \\
25 \cdot 5 \\
2 \cdot 7\end{array}$ & $\begin{array}{r}1 \cdot 7 \\
13 \cdot 9 \\
2 \cdot 9\end{array}$ & $\begin{array}{l}\text { N.S. } \\
1 \cdot 0 \\
\text { N.S. }\end{array}$ & Schizophrenia & $\overline{10 \cdot 3}$ \\
\hline
\end{tabular}

drawn from the wards and out-patient clinics of the Maudsley Hospital, and from the observation ward of St. Francis Hospital, Dulwich.

The remaining 116 patients were Army personnel who had broken down psychiatrically after their induction into the Service. All had been seen previously by more than one Army psychiatrist and all had been deemed sufficiently sick to be recommended for discharge on psychiatric grounds. All members of this military group were British "other ranks"; they comprised fifty anxiety states, 44 hysterics, and 22 cases of psychopathic personality.

The military patients were all males (age range: $18-46$, mean $22 \cdot 8$ years). The hospital patients included 37 female and 22 male depressives (age range: 19-75, mean 36.4 years), 22 female and 29 male schizophrenics (age range: 15-46, mean $27 \cdot 1$ years), four female and sixteen male cases of psychopathic personality (age range: $18-34$, mean $22 \cdot 1$ years), and ten female and sixteen male neurotics (age range: 14-60, mean $31 \cdot 1$ years).

CoNTROL Group. - The healthy controls represented a similar dichotomy of sampling. 152 of them were civilians: physicians, nurses, hospital workers, business men, and housewives, and the remaining 202 were healthy soldiers drawn from a neighbouring R.A. depot. 
The military group included recent inductees from all over the country, troops who had lately returned from overseas, and regular soldiers awaiting drafting to other units. The investigation was carried out in 1948-49, i.e. during the period of conscription but after the end of the war. The bias of self-selection was avoided since the soldiers did not volunteer for this investigation, but were detailed to report to the Institute of Psychiatry by special arrangement with the War Office.

All members of the military control groups were males (age range: 18-52, mean $25 \cdot 5$ years). Of the civilians, 63 were females and 89 males (age range: $16-68$, mean $31 \cdot 2$ years).

State of Health.- Since the investigation concerned the differential incidence of somatic disorders experienced by the subjects in the past, every effort was made to exclude subjects suffering from any of these disorders. Both the psychiatric and the control groups were free from any ascertainable bodily disease at the time of examination, though of course the same could not be said of their symptoms, for psychiatric patients are known to experience complaints referable to the soma, and such symptoms are not readily checked by reference either to the experience of relatives or to the medical record. It was therefore thought desirable sharply to distinguish bodily complaints from bodily diseases in the analysis of the results and to express the figures for each group separately. Even so it may be mentioned here that the incidence of unjustifiable and hypochondriacal exaggeration of symptoms appeared to be low in all patient groups by comparison with their previous history of actual illnesses.

\section{RESULTS}

These may be studied in detail in Table I. The statistical technique employed obeyed the strictures of Lewis and Burke (1949) concerning the $\chi^{2}$ method, in that the total expected incidence (actual incidence/total number) exceeded 0.24 for significance evaluation, and the differential contributory expected number had to be at least 10 . The modified $\chi^{2}$ analysis used (exemplified in detail in Table II) enabled the actual contribution to the total $\chi^{2}$ to be computed for each diagnostic group and sub-group,

TABLE II

Example of Method of Computing Differential $\chi^{2}$ Contribution of each Diagnostic Group

\begin{tabular}{|c|c|c|c|c|c|c|c|c|c|}
\hline \multirow{2}{*}{$\begin{array}{l}\text { Diagnostic } \\
\text { Group }\end{array}$} & \multicolumn{2}{|c|}{ Influenza } & \multicolumn{2}{|c|}{ Expected Number } & \multicolumn{2}{|c|}{$\begin{array}{l}\text { Deviation from } \\
\text { expected }\end{array}$} & \multicolumn{3}{|c|}{ Contribution to $\chi^{2}$} \\
\hline & $\begin{array}{c}\text { Actual } \\
\text { Incidence }\end{array}$ & $\begin{array}{c}\text { Non- } \\
\text { Incidence }\end{array}$ & Incidence & $\begin{array}{c}\text { Non- } \\
\text { Incidence }\end{array}$ & Incidence & $\begin{array}{c}\text { Non- } \\
\text { Incidence }\end{array}$ & Incidence & $\begin{array}{c}\text { Non- } \\
\text { Incidence }\end{array}$ & Total \\
\hline $\begin{array}{lr}\text { Controls } & (354) \\
\text { Psychopaths } & (42) \\
\text { Neurotics } & (120) \\
\text { Depressives } & (59) \\
\text { Schizophrenics } & (51)\end{array}$ & $\begin{array}{r}186 \\
16 \\
75 \\
58 \\
41\end{array}$ & $\begin{array}{r}168 \\
26 \\
45 \\
1 \\
10\end{array}$ & $\begin{array}{r}212 \cdot 75 \\
25 \cdot 24 \\
72 \cdot 12 \\
35 \cdot 46 \\
30 \cdot 65\end{array}$ & $\begin{array}{r}141 \cdot 25 \\
16 \cdot 76 \\
47 \cdot 88 \\
23 \cdot 54 \\
20 \cdot 35\end{array}$ & $\begin{array}{r}-26 \cdot 75 \\
-9 \cdot 24 \\
+\quad 2 \cdot 88 \\
+22 \cdot 54 \\
+10 \cdot 35\end{array}$ & $\begin{array}{l}+26 \cdot 75 \\
-9 \cdot 24 \\
-2 \cdot 88 \\
-22 \cdot 54 \\
-10 \cdot 35\end{array}$ & $\begin{array}{r}3 \cdot 37 \\
3 \cdot 38 \\
0 \cdot 12 \\
14 \cdot 32 \\
3 \cdot 48\end{array}$ & $\begin{array}{r}5 \cdot 07 \\
5 \cdot 09 \\
0 \cdot 18 \\
21 \cdot 58 \\
5 \cdot 26\end{array}$ & $\begin{array}{r}8 \cdot 44 \\
8 \cdot 47 \\
0 \cdot 30 \\
35 \cdot 90 \\
8 \cdot 74\end{array}$ \\
\hline Total Subjects (626) & 376 & 250 & $376 \cdot 22$ & $249 \cdot 78$ & 0 & 0 & $24 \cdot 67$ & $37 \cdot 18$ & $61 \cdot 85$ \\
\hline
\end{tabular}

Expected Incidence $=\frac{376}{626}=0 \cdot 601 ;$ Expected Non-Incidence $=\frac{250}{626}=0 \cdot 399 ;$ Total $\chi^{2}=61 \cdot 85 ; P<0 \cdot 001$

The outstanding history of influenza among the depressive population is clearly illustrated. Note that the contributions of the control and psychopath groups are negative and that the criteria of Lewis and Burke (1949) are fulfilled. 
and so indicated which particular group, if any, was outstanding in having a history of the disorder under investigation. In this connection it will be noted (Table II) that the deviations from the expected incidence may be either positive or negative, since it is just as significant that, for example, controls and psychopaths do not tend to have influenza in their medical histories, as it is that cases of depression do have such a history.

In general it may be seen from Table I that there is a higher incidence of physical illness and of complaints referable to the body in the medical histories of psychiatric patients than there is in a comparable sample of the general population. From the analysis of diagnostic group differential incidences, it can also be seen that this increased history loading is heavier in the case of the psychotics than it is in that of the neurotics or the character disorders. But over and above these gross differences (seen additionally in Table III in which total illness loadings are analysed by diagnostic

TABLE III

Analysis by Psychiatric Diagnosis of Total Somatic Illness History Loadings

\begin{tabular}{|c|c|c|c|c|c|}
\hline \multirow{2}{*}{$\begin{array}{l}\text { Psychiatric } \\
\text { Diagnosis }\end{array}$} & & \multicolumn{2}{|c|}{ Total Somatic Complaints } & \multicolumn{2}{|c|}{ Total Somatic Diseases } \\
\hline & & Mean & S.D. & Mean & S.D. \\
\hline $\begin{array}{l}\text { Controls ... } \\
\text { Psychopaths .. } \\
\text { Neurotics .. } \\
\text { Depressives . . } \\
\text { Schizophrenics }\end{array}$ & $\begin{array}{l}. \\
\because \\
\because \\
.\end{array}$ & $\begin{array}{r}1 \cdot 706 \\
2 \cdot 201 \\
3 \cdot 491 \\
8 \cdot 334 \\
10 \cdot 320\end{array}$ & $\begin{array}{l}1 \cdot 391 \\
3 \cdot 674 \\
6 \cdot 532 \\
2 \cdot 176 \\
3 \cdot 005\end{array}$ & $\begin{array}{r}6 \cdot 291 \\
6 \cdot 800 \\
8 \cdot 907 \\
13 \cdot 654 \\
13 \cdot 820\end{array}$ & $\begin{array}{l}1 \cdot 722 \\
4 \cdot 845 \\
2 \cdot 799 \\
4 \cdot 447 \\
5 \cdot 402\end{array}$ \\
\hline
\end{tabular}

Note that the number of both complaints and diseases increases with severity of the mental disorder.

group), there is the interesting revelation that certain groups of disorders occur in the histories of certain psychiatric diagnostic groups more often than in others. Thus, for example, depressed patients, prior to their psychiatric disturbance, would seem to have suffered from disorders referable to the gastro-intestinal tract (chronic appendicitis, peptic ulcer, haemorrhoids, dysentery, and "stomach troubles") not only more than controls but also more than neurotics or schizophrenics, while schizophrenics similarly have a higher somatic illness experience of disorders referable to the locomotor and cardiovascular systems (hypertension, muscular rheumatism, "back and neck pains") than any of the other diagnostic groups. It is, in fact, possible to draw up tables based on the figures from which Table I is derived, to show the positive correlations between differential psychiatric diagnosis and the history of specific bodily disorders in the past (Table IV, overleaf), and between such psychiatric states and the negative incidence of other bodily disorders (Table V, overleaf). Thus, for example, it can be seen that whereas a history of pertussis was found significantly in the medical illness loading of patients who subsequently developed both depressive and schizophrenic psychoses, this disease has a negative correlation with the later development of neuroticism. These tables extend the more limited yet similar findings of Ross and others (1950). 
TABLE IV

Outstanding Positive Correlations between Present Mental State and Differential Pattern of SPecific Complaints and Diseases' in Histories of Three TyPes of Psychiatric Disorder as COMPared With Medical Histories of the Control Group

No particular positive correlations existed for the psychopathic group

\begin{tabular}{|c|c|c|c|c|c|}
\hline \multicolumn{2}{|c|}{ Psycho-Neurosis } & \multicolumn{2}{|c|}{ Depression } & \multicolumn{2}{|c|}{ Schizophrenia } \\
\hline Symptoms & Diseases & Symptoms & Diseases & Symptoms & Diseases \\
\hline $\begin{array}{l}\text { Déjà vu } \\
\text { Palpitations } \\
\text { Dyspnoea }\end{array}$ & \begin{tabular}{l} 
Rheumatic \\
\multicolumn{1}{c}{ fever } \\
Head injury \\
Migraine \\
Syncope \\
Impacted \\
$\quad$ molars \\
Rubella \\
Pleurisy
\end{tabular} & $\begin{array}{l}\begin{array}{l}\text { Stomach } \\
\text { trouble }\end{array} \\
\text { Constipation } \\
\text { Indigestion } \\
\text { Gastric } \\
\text { stomach } \\
\text { Nasal catarrh } \\
\text { Pruritus } \\
\text { Blurred } \\
\text { vision }\end{array}$ & \begin{tabular}{l} 
Rickets \\
Influenza \\
Eczema \\
Peptic ulcer \\
Herpes zoster \\
Whooping \\
\multicolumn{1}{c}{ cough } \\
Neuritis \\
Colitis \\
Haemorrhoids \\
Dermatitis \\
Dysentery \\
Rubella \\
Chronic \\
appendicitis
\end{tabular} & 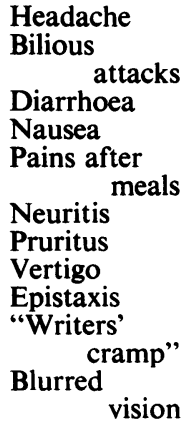 & $\begin{array}{l}\text { Whooping } \\
\quad \text { cough } \\
\text { Bronchitis } \\
\text { Boils } \\
\text { Fits } \\
\text { Muscular } \\
\quad \text { rheumatism } \\
\text { Rubella } \\
\text { Cardiovascu- } \\
\quad \text { lar disorders }\end{array}$ \\
\hline
\end{tabular}

TABLE V

Outstanding Negative Correlations between Present Mental State and Differential History Pattern of Specific Somatic Disorders AS COMPARED WITH CONTROLS

\begin{tabular}{l|c|c}
\hline Psycho-Neurosis & Depression & Schizophrenia \\
\hline Peptic ulcer & Nil & Peptic ulcer \\
Constipation & & \\
Diarrhoea \\
Haemorrhoids \\
$\begin{array}{l}\text { Eczema } \\
\text { Whooping cough } \\
\text { Dermatitis } \\
\text { Acne }\end{array}$ & & \\
\hline
\end{tabular}

INFLUENCE OF AGE AND SEX.-Both because of the reasonable assumption that the number of illnesses should increase with advancing years, and because of the low percentage of females in the control group as compared with a fairly even sex distribution in the disturbed psychotic groups, it is of urgent necessity to examine the exact contribution of both these variables to the data.

With regard to sex, it should be recalled that no specifically gynaecological complaint or disorder was included in the list investigated, though a differential sex incidence of many specific organic disturbances is, of course, known to occur. In order to smooth out these differences, the total loadings of complaints and diseases of the various psychiatric diagnostic groups (including the controls) were compared for possible sex distinction. A two-way analysis of variance was computed in which 
the illness experience of members of the control group and that of patients with neurosis, psychopathy, depression, and schizophrenia were compared, each variable being held separately constant to assess which contributed principally to the variance. Table VI gives the extent of significance of these differences and shows that the combination of diagnosis and sex accounts for 20.5 per cent. of the variance for complaints, and for $52 \cdot 1$ per cent. of actual somatic diseases, whereas psychiatric

TABLE VI

Results of a Paired Two-Way Analysis of Variance to assess Possible Influence of Age and SEX Variables as COMPaRed WITH EFfECT OF Psychiatric Diagnosis

\begin{tabular}{|c|c|c|c|c|c|c|c|}
\hline \multirow{2}{*}{$\begin{array}{c}\text { Incidence } \\
\text { of Somatic } \\
\text { Disorders }\end{array}$} & \multicolumn{4}{|c|}{ Combination of Factors Assessed } & \multirow{2}{*}{$\begin{array}{c}F \text { Ratio } \\
33 \cdot 543\end{array}$} & \multirow{2}{*}{$\begin{array}{c}\text { Significance } \\
.001\end{array}$} & \multirow{2}{*}{$\frac{\eta^{2}}{.521}$} \\
\hline & Diagnosis plus sex & . & . & .. & & & \\
\hline \multirow{3}{*}{ Complaints } & Sex only & . & . & .. & $1 \cdot 308$ & N.S. & .005 \\
\hline & Diagnosis only & . & . & . & $76 \cdot 000$ & .001 & $\cdot 518$ \\
\hline & Addition of sex to & liagr & & . & $2 \cdot 487$ & N.S. & - \\
\hline \multirow{4}{*}{ Diseases } & Diagnosis plus sex & . & . & .. & $7 \cdot 959$ & .001 & $\cdot 205$ \\
\hline & Sex only & . & . & . & $3 \cdot 386$ & N.S. & .012 \\
\hline & Diagnosis only & . & .. & . & $16 \cdot 043$ & .01 & $\cdot 185$ \\
\hline & Addition of sex to & liagr & & . & $1 \cdot 379$ & N.S. & - \\
\hline \multirow{4}{*}{ Complaints } & Diagnosis plus age & . & . & . & $11 \cdot 174$ & .001 & $\cdot 232$ \\
\hline & Age only & $\cdots$ & . & . & $4 \cdot 913$ & .01 & .023 \\
\hline & Diagnosis only & . & . & . & $40 \cdot 224$ & .001 & $\cdot 226$ \\
\hline & Addition of age to & diagr & & . & $<1$ & N.S. & - \\
\hline \multirow{4}{*}{ Diseases } & Diagnosis plus age & . & .. & . & $41 \cdot 461$ & $\cdot 001$ & $\cdot 529$ \\
\hline & Age only & . & .. & .. & $13 \cdot 127$ & .001 & .059 \\
\hline & Diagnosis only & . & .. & .. & $142 \cdot 590$ & $\cdot 001$ & $\cdot 508$ \\
\hline & Addition of age to & liagı & sis & . & $2 \cdot 249$ & .05 & - \\
\hline
\end{tabular}

diagnosis alone accounts for 18.5 per cent. and 51.8 per cent. of the variance respectively. The additional variance obtained by adding the sex variable to that of diagnosis was not statistically significant either for complaints or for diseases.

So far as age is concerned, the matter is slightly more complicated, for the neurotics and psychopaths as a group were younger than any of the other diagnostic categories. A similar statistical procedure was however undertaken, a two-way analysis of variance being run on four diagnostic groups (controls, neurotics plus psychopaths, depressives, and schizophrenics) whose separate totals of somatic complaints and 
diseases were set out in three age ranges: 15-24, 25-34, and 35-44. As might have been expected, the mean numbers of total complaints and diseases increased progressively as age advanced, but the figures showed that they did so more rapidly in the psychotics than in the controls. As with the variable of sex, while diagnosis and age factors accounted for 23.2 per cent. of the variance with respect to complaints and for 52.9 per cent. with respect to somatic discases (Table VI), 22.6 per cent. and 50.8 per cent. of this respectively was due to psychiatric diagnosis alone and the addition of age to diagnosis yielded an $F$ ratio which had no statistical significance.

It can therefore be stated that, under the conditions of this investigation, neither the factor of age (within the adult range of 15-44 years), nor that of sex, bore any significance to the somatic illness experience of the subjects assessed relative to the importance of the psychiatric diagnostic classifications in which they were placed.

Estimates of VALIDITY AND Reliability.-It is recognized that the findings herein reported, since they are relatively new to psychiatric experience, will require stern tests of their validity. In view of the paucity of similar data elsewhere in the literature, however, it is difficult to assess the results of the present research in terms of strict scientific validity; but it is obvious that if our results are to measure up to certain studies of isolated somatic-disease epidemiology in a psychiatric population, then our control data must reflect reasonably accurately the historical morbidity experience of a representative sample of the British population. It is perhaps unfortunate that such a nation-wide survey does not exist at present. The Registrar-General's annual statistical report relates mainly to mortality figures, and even the incidence of such a widespread notifiable infection as tuberculosis is given either in terms of mortality, or of numbers of notifications by district, or, in other reports, of the relative infectivity of the human or bovine forms or of the radiological incidence of minimal lesions. The writer has not found a recent reference to the historical incidence of the number of individuals who have actually developed tuberculosis as an illness over a wide area of the country. Our percentage of 1.9 represents a much lower incidence than that of minimal lesions discovered by mass miniature radiography, and a much higher incidence than the general mortality figures for this disease.

A more comforting indication of the internal reliability of our data is the relative incidence of the acute zymotic infections of childhood. Table I shows that there are no significant differences between the control group and the various psychiatric groups in the historical incidence of measles, mumps, chicken-pox, and scarlet fever. Furthermore the medical history incidence of 74.9 per cent. in the case of measles for the control group is practically identical with that of the Berkeley workers (Bayer and Snyder, 1950), who reported an incidence of 78 per cent. in their longitudinal study of 126 "normal" children followed periodically up to the age of 18 years. Allowing for obvious cultural, climatic, and geographical differences, it is perhaps surprising that the figures for other childhood disorders agree so well. Chicken-pox (71 per cent. as against 42 per cent. in Great Britain) shows considerable discrepancy, but mumps (48 per cent. against 43 per cent.) agrees well, as do rubella (18 per cent. 
against 12 per cent.) and diphtheria ( 4 per cent. against 7 per cent.). Before leaving the Berkeley report, it is interesting to compare figures for other disorders which are close to our own. Thus, for example, the incidence of appendicectomy was 15 per cent. in their study, 14 per cent. in ours; fractures 35 per cent. against 20 per cent. ; coughs and colds 67 per cent. against our 52 per cent. Bearing in mind that the two groups studied differed in many respects, were of a different age range, and were many thousands of miles apart, the degree of concordance between the figures is remarkable.

That the figures in the present survey were obtained largely from questionnaire study appears to require some justification, since this method of investigation has been open to considerable criticism in the past. It is of significance however that the morbidity survey conducted by Stocks on behalf of the Ministry of Health (1945) employed a similar methodology of self-assessment and that its validity compared well with non-questionnaire surveys. Analysis of the subjects' statements concerning their health over the previous 3 months revealed that 11 per cent. of the men and 15 per cent. of the women had suffered from nervous or functional disabilities - an incidence practically identical with that reached by Fraser (1947) after very thorough investigation of a group of factory workers. The same report by Fraser's Medical Research Council team contains other interesting comparisons with our findings. For example, his figures (p. 27) show that in both men and women age has relatively little influence on the incidence of either neurotic or physical illness in the range 21-60 years, and also (p. 25) that subjects found to have neurotic illness in industry, not only have a heavier previous history of neurotic breakdowns but also a heavier loading of previous physical illnesses. The M.R.C. Report reveals that $29 \cdot 6$ per cent. of neurotic males and 30.6 per cent. of neurotic females had had previous severe physical illness, while only 20.1 per cent. sympton-free males and 16.8 per cent. symptom-free females had suffered in this way. Both these findings are unexpected, but both are in accord with the results reported here.

As regards the actual differential incidence of various somatic complaints and disorders among the psychiatric groups as opposed to the controls, there are many indications that our findings match the experience of other writers. Thus Wolff (1948) mentions that 8 per cent. of inductees into the U.S. Army complained of recurrent severe headaches, as against 50 per cent. of neurotic soldiers. Our figures (Table I) record percentages of $7 \cdot 2$ and $42 \cdot 3$ respectively for this symptom. Another type of headache, the incidence of which has been accurately determined in an American population, is migraine. Lennox (1941) gives the incidence of migraine in 1,000 otherwise healthy medical students and nurses as $5 \cdot 4$ per cent. Our figure of $8 \cdot 2$ per cent. in the control group compares reasonably well, especially as the comparable incidence among cases of psychoneurosis, anxiety state, is so widely different at $37 \cdot 7$ per cent.

A report of the Ministry of Health (1922) on the incidence of rheumatic diseases mentions that 2.76 per cent. of the total list strength attended their practitioners for "rheumatic disease" in 1922. This is a vague diagnosis, but our control figures for certain forms of non-articular rheumatism agree well with this estimate (e.g. lumbago 
2 per cent.; fibrositis 3 per cent.). The results when psychiatric patients' histories are considered are very different. Harris (1938) in a 10 to 12-year follow-up study of 259 cases of neurosis, anxiety state, found that 10 per cent. of 98 cases available for study had suffered from muscular rheumatism, an estimate which may be compared with our figure of 12 per cent. for controls and 18 per cent. for anxiety states. Harris also found 6 per cent. of his cases with essential hypertension (present series: 5 per cent. anxiety states, 10 per cent. controls); 4 per cent. with arthritis (present series: 3 per cent. anxiety states, 5 per cent. controls); and 21 per cent. with gastric symptoms ("gastric stomach" in present series: anxiety states 22 per cent., controls 3 per cent.).

Finally, it is relevant to refer to another study (Eysenck, 1947) in which the incidence of somatic complaints in cases of anxiety state is compared with that in cases of hysteria. Analysis of responses to a psychological test for "neuroticism" (Maudsley Medical Questionnaire) showed that anxiety-state cases answered "yes" more often than hysterics in most cases. The same tendency was present in our series. Out of 45 comparable disorders, anxiety states showed a higher percentage incidence in 32 , as against hysterics who showed a higher percentage incidence in thirteen.

\section{Discussion}

The high incidence of somatic complaints in psychiatric patients has been referred to by several authors, including Billings (1939) and Hemphill (1939), but the efforts of Ross and others (1950) to assess the concomitance of mental disorder and such somatic vegetative diseases as peptic ulcer, asthma, diabetes mellitus, rheumatoid arthritis, and hypertension, lead to the conclusion that none of these disorders presented more commonly among the population of a Canadian mental hospital than in the general population at risk outside it. For purposes of comparison with our present findings, however, it is significant that the Canadian workers were evaluating the concurrence of mental ánd physical disease, and that, in so far as their findings included observations that peptic ulcer and rheumatoid arthritis at least were less common among psychotics than among the general population, their results are in accord with our thesis. The conclusions regarding rheumatoid arthritis confirm those of Gregg (1939). It appears that the response to stress involves the total personality: the sphere of major response may be somatic, with resulting physical disease; it may be psychological, and followed by psychiatric disturbance; or it may partake of both with the development of both somatic and psychic disorders, either together or in alternation.

The literature on immunity has concerned itself little with these considerations, yet certain indications that a case can be made out for the implication of psychological factors in disease susceptibility and host resistance are beginning to appear. A recent paper by Vaughan (1949) testifies to a decreased ability on the part of 22 schizophrenic subjects to develop antibody titres to inoculated pertussis vaccine antigen as compared with seven control subjects. Not only were the maximal titres of the schizophrenic group significantly lower than those of the controls, but they also showed a differential distribution among the various sub-types of schizophrenia studied, paranoid and better-integrated schizophrenics giving relatively higher titres than constitutionally 
determined and less well-integrated hebephrenics. That these findings are not peculiar to the inoculation of pertussis vaccine or to a schizophrenic population has already been shown (Lovett Doust and Chappell, unpublished observations). Similar experiments were performed using the $\mathrm{H}$ and $\mathrm{Vi}$ antigens of the Lister Institute T.A.B. vaccine on more acute and less chronic institutionalized schizophrenics and also on patients with other psychiatric disorders. In schizophrenia, lower absolute titres were found than in controls; in affective disorder, depressive type, the titres were higher but still not up to control levels; and in cases of psychoneurosis, initial postinoculation titres were equivalent to those in controls, but were ill-sustained and fell off markedly during a 10-week observation period.

If such antibody responses can be held to represent certain aspects of the immunity threshold, then it may be said that psychiatric variables are at least of equal importance with those dependent upon adequate nutrition, since Gell (1948), studying titres obtained after the inoculation of starved Wuppertal Concentration Camp prisoners in Germany with a complex group of provocative antigens, found differences between the results from this group and from healthy, well-nourished British soldiers which only just reached the level of statistical significance. In view of these figures, Gell was forced to the conclusion that even gross, pre-mortal undernutrition "does not play as large a part in widespread epidemics as is generally supposed", and hence that the "psychological imponderables" may be assumed to be a responsible influence. These psychological imponderables might also go a long way to explain the wide variations in susceptibility which exist among healthy individuals, a fact which still puzzles immunologists (Beattie and Dickson, 1943).

Alternation of Psycho-Somatic Diseases.-It appears to the writer an attractive hypothesis that a man's individual constitution is a limiting factor with respect to the degree of stress which he can withstand. If it may be assumed that breakdown follows exposure to stresses which go beyond the individual's threshold of tolerance, then not only is it logical to include in such stresses their emotional and psycho-dynamic components, but it is also just as likely that the resulting total personality breakdown may predominantly affect the psyche as it is that the soma may be principally involved. Disease and disorder may conveniently and with some truth be thought of as homeostatic defences against the onslaught of stress. The schizophrenic's withdrawal from intolerable stress into a dream world of relative unawareness is, at least temporarily, as successful for him as it is socially morbid for his place in society; the "mentally stable" patient who is incapacitated by acute stress and spends a month away from his work with pneumonia, and the recurrent invalidism necessitated by the chronically summating stresses leading to recurrent exacerbations of rheumatoid arthritis, are equally morbid in so far as they solve a temporary situation on a basis of somatic pathology which often becomes permanent and irreversible.

Mental and physical diseases alike are both pathways of reaction and defences against stress. The greater the mental stability and balance, the more likely is the psychiatric pattern of breakdown to be chosen in preference to the "canalization" and 
"somatization" of breakdown into tissue and organ systems. The psychiatric investigation of cases of somatic disease (e.g. Alexander, 1950) shows that under such somatic defences there lie conflicts and profiles which are also common in many respects to specific organic disease conditions, and Appel and Rosen (1950) have illustrated the alternation from psychiatric to somatic disturbance and vice versa which is obvious in so far as it is looked for. Indeed it may be said with Hippocrates (Aphorisms, VII, 5 and VI, 21) that the one orientation of disease often acts as a protection against the other. Clow and Prout (1946) have recently reported amelioration of mental disorder in cases in which intercurrent physical disorder appeared. Of their 100 unselected mental hospital patients who experienced a physical illness during their period of hospitalization, they found that 67 improved and eleven recovered completely.

Our previous findings suggest that, since the medical histories of psychiatric patients show a greater loading with somatic disease than do those of a comparable healthy control group, then either their experience of stress has been greater or their resistance to it is intrinsically lower than that of the controls. Probably both factors operate and, since initial breakdown predisposes to other breakdowns, we may assume a vicious-circle mechanism.

Physical Disease the Mask of Psychosis.-A further implication of these results lies in the fact that, in historical sequence, it is usually the physical illness mechanism of defence which is first employed by the subject under stress. That psychiatric breakdown is a last defence, resorted to when other means have failed, would seem to be a function of the degree of emotional stability and maturity achieved by the subject (Draper and others, 1946). Psychiatric investigation of apparently mentally stable patients with such psychosomatic diseases as asthma, essential hypertension, and peptic ulcer, reveals a chaos of repressed and unconscious conflicts, tensions, and desires for which the organic disease is often an obvious symbolic means of expression and of partial detumescence. Too rapid and dramatic a removal of somatic symptoms (e.g. by ACTH in rheumatoid arthritis) not uncommonly leads to a concomitant psychiatric disturbance or fully-fledged psychosis. It is suggested therefore that the physical illness, determined as it is by the combination of type of stress and personality pattern, is both the mask and the means of expression for the tensions and conflicts which, if uncanalized and unexpressed, would amount to a psychosis.

\section{SUMmaRY}

(1) The physical illness history, in terms of 38 bodily complaints and 72 somatic diseases, was assessed in 354 healthy adult controls and in 272 patients with a variety of psychiatric disorders.

(2) It was found that the physical illness experience of the psychiatric patients was significantly in excess of that of the controls, psychotics having a heavier loading of previous physical illness than neurotics or psychopaths. The influence of age and sex was insignificant in comparison with that of psychiatric diagnosis.

(3) The childhood illness experience in all groups did not differ significantly with respect to most zymotic diseases. A differential pattern of organ system reaction was 
found to be present for other disorders. The somatic illness history of neurotics included many examples of minor physical disability, that of depressives showing especially a loading with gastro-intestinal disorders, and that of schizophrenics a loading with locomotor and cardio-vascular diseases.

(4) The findings are discussed with reference to their reliability and validity, and their immunological implications considered from the psychiatric point of view.

My thanks are due to Professor Aubrey Lewis, M.D., F.R.C.P., Head of the Department of Psychiatry, University of London Institute of Psychiatry, for facilities enabling this work to be carried out; to the War Office, Lt.-Col. H. Posner, M.C., R.A.M.C., and Col. Bootle-Wilbraham, D.S.O., M.C., for the provision of military personnel for investigation; to Mr. A. Lubin, Ph.D., statistical psychologist to this Institute and Mr. J. Feeley, B.A., of the New York Hospital, Cornell Medical Centre, for statistical advice and computational assistance respectively. I also gladly acknowledge the co-operation of my colleagues at the Bethlem Royal and Maudsley Hospitals in supplying patients for study and the help readily given by Dr. Mary Pringle and others in supplementing the control group. Finally I should like to thank the Trustees of the Nuffield Foundation for their interest in this research and for their award of a Medical Fellowship enabling it to be carried out.

\section{REFERENCES}

Alexander, F. (1950). "Psychosomatic Medicine." Norton, New York.

Appel, J., and Rosen, S. R. (1950). Psychosomat. Med., 12, 236.

Balch, H. H. (1950). J. Immunol., 64, 397.

Bayer, L. M., and Snyder, M. M. (1950). Child Development, 21, 93.

Beattie, J. M., and Dickson, W. E. C. (1943). "Textbook of Pathology", p. 124, 4th ed. Heinemann, London. Billings, E. (1939). "Handbook of Elementary Psychobiology and Psychiatry." Macmillan, New York.

Boycott, A. E., and Price-Jones, C. (1926). J. Path. Bact., 29, 87.

Brodman, K., Mittelmann, B., Wechsler, D., Wieder, A., and Wolff, H. G. (1947a). Psychosomat. Med., 9, 37. $-\ldots-$ - (1947b). Ibid., 9, 45.

Clow, H. E., and Prout, C. T. (1946). Amer. J. Psychiat., 103, 179.

Dougherty, T. F., Chase, J. H., and White, A. (1945). Proc. Soc. exp. Biol. N. Y., $58,135$.

Draper, G., Dupertuis, C. W., and Caughey, J. L., Jr. (1944). "Human Constitution in Clinical Medicine", p. 72. Hoeber, New York and London.

Eysenck, H. J. (1947). “Dimensions of Personality." Kegan Paul, London.

Fraser, R. (1947). Med. Res. Council Ind. Hlth. Res. Board. Rep. No. 90, pp. 25 and 27. H.M. Stationery Office, London.

Gell, P. G. H. (1948). Proc. roy. Soc. Med., 41, 323.

Gregg, D. (1939). Amer. J. Psychiat., 95, 853.

Halliday, J. L. (1948). "Psychosocial Medicine". Heinemann, London.

Harris, A. (1938). Brit. med. J., 2, 649.

Hemphill, R. (1939). J. ment. Sci., 85, 119.

Hoagland, H., and Pincus, G. (1950). In "Proc. First Clinical ACTH Conference", ed. J. R. Mote, p. 544. Blakiston Company, Philadelphia.

Holmes, T. H., Goodell, H., Wolf, S., and Wolff, H. G. (1950). The Nose: an experimental study of reactions within the nose in human subjects during varying life experiences". Thomas, Springfield, Ill.

Lennox, W. G. (1941). "Science and Seizures". Harper, New York.

Lewis, D., and Burke, C. J. (1949). Psychol. Bull., 46, 433.

Lovett Doust, J. W. (1952). J. ment. Sci. In the Press. and Chappell, P. Unpublished observations.

Ministry of Health. (1924). Report No. 23. H.M.S.O., London.

Ministry of Health. (1945). Mon. Bull. Min. Hlth., 4, 119.

Phillips, R. J. (1937). Brit. med. J., 2, 363.

Ross, W. D., Hay, J., and McDowall, M. F. (1950). Psychosomat. Med., 12, 179.

Selye, H. (1950). "Stress". Acta Inc., Montreal.

Vaughan, W. T., Jr., Sullivan, J. C., and Elmadjian, F. (1949). Psychosomat. Med., 11, 327.

White, A., and Dougherty, T. F. (1946). Ann. N. Y. Acad. Sci., 46, 859.

Wilson, G. S., and Miles, A. A. (1946). "Topley and Wilson's Principles of Bacteriology and Immunity", 3rd ed., vol. 2, p. 1217. Arnold, London.

Wolff, H. G. (1948). "Headache and Other Head Pain”. Oxford Univ. Press, New York. 